\title{
Closed-Form Performance of MFSK Signals with Diversity Reception over Non-identical Fading Channels
}

\author{
Le Cao, Meixia Tao and Pooi Yuen Kam \\ Department of Electrical and Computer Engineering \\ National University of Singapore, Singapore 119260 \\ Email: $\{$ caole, mxtao, elekampy\}@nus.edu.sg
}

\begin{abstract}
This paper provides a comprehensive study on the error performance of noncoherent orthogonal $M$-ary frequencyshift-keying (FSK) signals with various diversity combining schemes over fading channels. The diversity branches are assumed to be independent and non-identically distributed (i.n.d) Rayleigh fading. Closed-form expressions for the symbol error probability with an arbitrary diversity order and any modulation level are obtained for optimal combining (OC), equal gain combining (EGC) and log-likelihood ratio based selection combining (SC-LLR). Our analytical results show that EGC performs closely to OC over slightly unbalanced channels, whereas for highly unbalanced channels SC-LLR performs more closely to OC than EGC.
\end{abstract}

\section{INTRODUCTION}

Diversity reception is a powerful technique in wireless communications that reduces the depth of the fades and/or the fade duration by supplying the receiver with multiple replicas of the transmitted signal through independent fading channels. The combining schemes in diversity reception are sophisticated and include maximum ratio combining, equal gain combining and selection combining, thereby enabling a flexible tradeoff between error performance and implementation complexity. Performance analysis of modulation techniques over fading channels with diversity reception is a steady research topic in the literature. As in many applications the random channel amplitudes and carrier phases cannot be tracked accurately, noncoherent frequency-shift-keying (FSK) technique has gained much attention. The data information of FSK signals is carried in the frequency and thus it allows the square-law detector which can eliminate the acquisition for channel amplitudes and phases. In addition, the FSK signals can be designed to be orthogonal so as to minimize the error probability.

The performance of noncoherent orthogonal FSK over independent and non-identically distributed (i.n.d) Rayleigh fading channels with optimal combining (OC) and equal gain combining (EGC) were derived in [1] and [2], respectively. Note that these results are for binary signals or for dual diversity only. In [3], [4], the authors evaluated the symbol error probability (SEP) of noncoherent orthogonal $M$-ary FSK (MFSK) signals over independent and identically distributed (i.i.d) and i.n.d fading channels respectively with traditional selection combining schemes, such as signal-to-noise ratio selection combining
(SC-SNR), signal-plus-noise selection combining (SC-S+N), and maximum output selection combining (SC-MO). Recently, a new selection combining scheme based on log-likelihood ratio, referred to as SC-LLR, was introduced in [5] and [6]. The authors therein also obtained the error performance of MFSK but the channels were assumed to be i.i.d only.

In this paper, we provide a comprehensive study on the performance of noncoherent orthogonal FSK signals over i.n.d Rayleigh fading channels with all aforementioned diversity combining schemes. Specifically, closed-form expressions for SEP of $M$-ary FSK signals with OC, EGC and SC-LLR are obtained. Compared with existing results in the literature, our contribution lies in the following. We generalize the performance study of FSK signals with the OC and EGC combining schemes from the binary signals and/or dual diversity case to the $M$-ary signals with arbitrary number of diversity branches, and the performance study of MFSK with SC-LLR from i.i.d channels to i.n.d channels. Furthermore, the comparison in both performance and complexity of different combining schemes when there exist different levels of unbalance between the diversity branches is firstly made.

The rest of this paper is organized as follows. In Section II, we describe the system model. Then, the symbol error probabilities for OC, EGC and SC-LLR are derived in Section III, IV and V, respectively. Section VI presents numerical results and discussions. Finally, this paper is concluded in Section VII.

\section{SYSTEM MODEL}

We consider a communication system in which an orthogonal $M$-ary FSK signal is transmitted through $L$ i.n.d diversity branches. The channel on each diversity branch is assumed to be slowly time-varying and frequency-nonselective Rayleigh fading. It introduces attenuations and phase shifts on the transmitted signal in conjunction with additive white gaussian noise (AWGN). The baseband received signal on the $l$ th branch at time $t$ can be modeled as

$$
r_{l}(t)=\alpha_{l} \tilde{S}_{k}(t) e^{j \theta_{l}}+\tilde{N}_{l}(t), \quad l=1,2, \ldots, L,
$$

where $\tilde{S}_{k}(t)$ is the $k$ th complex baseband signal, $\left\{\alpha_{l}\right\}_{l=1}^{L}$ and $\left\{\theta_{l}\right\}_{l=1}^{L}$ are the random Rayleigh distributed channel 
amplitudes and uniformly distributed phases over $[0,2 \pi]$ respectively, and $\left\{\tilde{N}_{l}(t)\right\}_{l=1}^{L}$ is a set of independent complex AWGN processes with power spectra density (PSD) $N_{0}$. The baseband MFSK signal waveforms are given by

$$
\tilde{S}_{k}(t)=\sqrt{\frac{E_{s}}{T_{s}}} e^{j 2 \pi f_{k} t}, \quad k=1,2, \ldots, M
$$

and they are orthogonal when the tone frequencies $\left\{f_{k}\right\}$ are chosen so that $\left|f_{k+1}-f_{k}\right|=1 / T_{s}$, where $E_{s}$ is the symbol energy and $T_{s}$ is the symbol duration. We assume that the $M$ signals are equiprobable.

In square-law detection, the received signal from each diversity branch is passed through a bank of matched filters followed by square-law envelop detectors. The outputs of each square-law envelop detector for different diversity branches are then combined using a certain criterion to form a decision metric. Let $y_{k l}$, for $k=1, \ldots, M$ and $l=1, \ldots, L$ denote the output of the $k$ th matched filter on the $l$ th diversity branch. It can be considered as the complex cross correlation between the received signal on the $l$ th branch $r_{l}(t)$ and the $k$ th possibly transmitted signal $\tilde{S}_{k}(t)$. Given that $\tilde{S}_{m}(t)$ is transmitted, $y_{k l}$ can be written as [7]

$$
y_{k l}= \begin{cases}E_{s} \alpha_{l} e^{j \theta_{l}}+\int_{0}^{T_{s}} \tilde{N}_{l}(t) \tilde{S}_{m}^{*} d t & k=m \\ \int_{0}^{T_{s}} \tilde{N}_{l}(t) \tilde{S}_{k}^{*} d t & k \neq m,\end{cases}
$$

where notation $*$ denotes the complex conjugate. In the following sections, we shall present the decision metrics and performances of different combining schemes.

\section{SEP OF OPTIMUM COMBINING}

In this section, we derive the SEP of noncoherent orthogonal MFSK signals with optimum combining. The idea here is to extend the results in [1] to $M$-ary FSK with arbitrary diversity orders.

In the OC scheme, the receiver computes the set of $a$ posteriori probabilities

$$
P\left(\tilde{S}_{k}(t) \mid\left\{r_{l}(t)\right\}_{l=1}^{L}\right), \quad k=1, \ldots, M
$$

and decides in favor of the message that has the largest value. The decision metric becomes [1, (3)]

$$
\Lambda_{k}=\sum_{l=1}^{L} \frac{\bar{\gamma}_{l}}{1+\bar{\gamma}_{l}}\left|y_{k l}\right|^{2}, \quad k=1, \ldots, M,
$$

where $\overline{\gamma_{l}}=\frac{\Omega_{l} E_{s}}{N_{0}}\left(\Omega_{l} \triangleq E\left[\alpha_{l}^{2}\right]\right)$ is the average SNR on the $l$ th branch and is assumed to be distinct for different l's. From (3) we can see that the optimal combining applies average SNRdependent weights to the outputs of square-law detectors.

Assuming that $\tilde{S}_{1}(t)$ is transmitted, the decision metric can be obtained by substituting (2) with $m=1$ into (3) as

$$
\begin{aligned}
& \Lambda_{1}=\sum_{l=1}^{L}\left\{\left(E_{s} \alpha_{l}{ }^{\prime} \cos \theta_{l}+Z_{1 l}^{R}\right)^{2}+\left(E_{s} \alpha_{l}{ }^{\prime} \sin \theta_{l}+Z_{1 l}^{I}\right)^{2}\right\} \\
& \Lambda_{k}=\sum_{l=1}^{L}\left\{\left(Z_{k l}^{R}\right)^{2}+\left(Z_{k l}^{I}\right)^{2}\right\}, \quad k=2, \ldots, M
\end{aligned}
$$

In (4) and (5), we have

$$
\begin{aligned}
\alpha_{l}{ }^{\prime} & =\sqrt{\frac{\bar{\gamma}_{l}}{1+\bar{\gamma}_{l}}} \alpha_{l} \\
Z_{k l}^{R} & =\operatorname{Re}\left\{\sqrt{\frac{\bar{\gamma}_{l}}{1+\bar{\gamma}_{l}}} \int_{0}^{T_{s}} \tilde{N}_{l}(t) \tilde{S}_{k}^{*}(t) d t\right\} \\
Z_{k l}^{I} & =\operatorname{Im}\left\{\sqrt{\frac{\bar{\gamma}_{l}}{1+\bar{\gamma}_{l}}} \int_{0}^{T_{s}} \tilde{N}_{l}(t) \tilde{S}_{k}^{*}(t) d t\right\} .
\end{aligned}
$$

Sets $\left\{E_{s} \alpha_{l}{ }^{\prime} \cos \theta_{l}+Z_{1 l}^{R}\right\}_{l=1}^{L}$ and $\left\{E_{s} \alpha_{l}{ }^{\prime} \sin \theta_{l}+Z_{1 l}^{R}\right\}_{l=1}^{L}$ are mutually independent, and each set consists of $L$ independent, Gaussian random variables, each with mean zero and variance $E_{s} N_{0} \bar{\gamma}_{l} / 2$ that depends on $l$. Similarly, for $\left\{Z_{k l}^{R}\right\}_{k=1}^{M}$ and $\left\{Z_{k l}^{I}\right\}_{k=1}^{M}$, each of the underlying Gaussian random variables has mean zero and variance $E_{s} N_{0} \bar{\gamma}_{l} / 2\left(1+\bar{\gamma}_{l}\right)$. Thus, the $l$ th summation term of $\Lambda_{k}$ for $k=1, \ldots, M$ has an exponential distribution. By using the characteristic function approach, the probability density function (PDF) of $\Lambda_{k}$ is shown as [1]

$$
\begin{aligned}
& p_{\Lambda_{1}}(\Lambda)=\sum_{l=1}^{L} \frac{B_{l}}{m_{l}} e^{-\frac{\Lambda}{m_{l}}} \\
& p_{\Lambda_{k}}(\Lambda)=\sum_{l=1}^{L} \frac{B_{l}{ }^{\prime}}{m_{l}{ }^{\prime}} e^{-\frac{\Lambda}{m_{l^{\prime}}}} \quad k=2, \ldots, M
\end{aligned}
$$

In (7) and (8), we have

$$
\begin{aligned}
B_{l} & =\prod_{i=1, i \neq l}^{L} \frac{\bar{\gamma}_{l}}{\bar{\gamma}_{l}-\bar{\gamma}_{i}} ; & B_{l}{ }^{\prime} & =\prod_{i=1, i \neq l}^{L} \frac{\bar{\gamma}_{l}\left(1+\bar{\gamma}_{i}\right)}{\bar{\gamma}_{l}-\bar{\gamma}_{i}} \\
m_{l} & =E_{s} N_{0} \bar{\gamma}_{l} ; & m_{l}{ }^{\prime} & =E_{s} N_{0} \bar{\gamma}_{l} /\left(1+\gamma_{l}\right) .
\end{aligned}
$$

Since the $M$ signals are equiprobable, the average symbol error probability can be written as

$$
P_{s}(e)=1-P\left\{\left\{\Lambda_{k}\right\}_{k=2}^{M}<\Lambda_{1}\right\} .
$$

By substituting the PDFs of $\Lambda_{1}$ and $\Lambda_{k}$ for $k=2, \ldots, M$ into (9) the average SEP of orthogonal MFSK with optimum combining for $L$ diversity branches is shown below

$$
\begin{aligned}
P_{s}(e)= & \sum_{m=1}^{M-1}\left(\begin{array}{c}
M-1 \\
m
\end{array}\right)(-1)^{m+1} \sum_{n_{1}, n_{2}, \ldots n_{L}} \frac{m !}{n_{1} ! n_{2} ! \ldots n_{L} !} \\
& \times\left(\prod_{i=1}^{L}\left(B_{i}{ }^{\prime}\right)^{n_{i}}\right) \sum_{l=1}^{L} \frac{B_{l}}{1+\bar{\gamma}_{l}\left(m+\sum_{i=1}^{L} \frac{n_{i}}{\bar{\gamma}_{i}}\right)} .
\end{aligned}
$$

In obtaining (10), the multinomial theorem is applied, i.e.

$\left(x_{1}+x_{2}+\ldots+x_{L}\right)^{m}=\sum_{n_{1}, n_{2}, \ldots n_{L}} \frac{m !}{n_{1} ! n_{2} ! \ldots n_{L} !} x_{1}^{n_{1}} x_{2}^{n_{2}} \ldots x_{L}^{n_{L}}$,

where the summation is taken over all multi-indices $\left(n_{1}, \ldots n_{L}\right) \in \mathbb{N}^{L}$ that sum to $m$. It can be verified that by letting $L=2$, the expression of (10) reduces to the dual diversity case in $[1,(18)]$, and by letting $M=2$, the result in (10) reduces to the binary signal in $[1,(8)]$. 


\section{SEP OF EGC SCHEME}

In this section, we analyze the performance of MFSK with the EGC combining scheme. Note that for the case of binary FSK, the SEP was obtained in [2] while the result for dual diversity was shown in [1].

In the EGC scheme, the outputs from the correlators are combined with equal weights. Thus, the decision metric can be obtained by ignoring the $\bar{\gamma}_{l} /\left(1+\bar{\gamma}_{l}\right)$ term in (3) as

$$
\Lambda_{k}=\sum_{l=1}^{L}\left|y_{k l}\right|^{2}, \quad k=1, \ldots, M .
$$

The derivation for the performance of EGC is similar to that of OC discussed in Section III. Given that $\tilde{S}_{1}(t)$ is transmitted, substituting (2) with $m=1$ into (11) leads to

$$
\begin{aligned}
& \Lambda_{1}=\sum_{l=1}^{L}\left\{\left(E_{s} \alpha_{l} \cos \theta_{l}+Z_{1 l}^{R}\right)^{2}+\left(E_{s} \alpha_{l} \sin \theta_{l}+Z_{1 l}^{I}\right)^{2}\right\} \\
& \Lambda_{k}=\sum_{l=1}^{L}\left\{\left(Z_{k l}^{R}\right)^{2}+\left(Z_{k l}^{I}\right)^{2}\right\}, \quad k=2, \ldots, M,
\end{aligned}
$$

where

$$
\begin{aligned}
& Z_{k l}^{R}=\operatorname{Re}\left\{\int_{0}^{T} \tilde{N}_{l}(t) \tilde{S}_{k}^{*}(t) d t\right\} \\
& Z_{k l}^{I}=\operatorname{Im}\left\{\int_{0}^{T} \tilde{N}_{l}(t) \tilde{S}_{k}^{*}(t) d t\right\} .
\end{aligned}
$$

The $l$ th summation term of $\Lambda_{1}$ has an exponential distribution, where the underlying Gaussian random variables have variance $E_{s} N_{0}\left(\bar{\gamma}_{l}+1\right) / 2$. On the other hand, $\Lambda_{k}$ for $k=2, \ldots, M$ is central chi-square distributed with $2 L$ degrees of freedom, since $Z_{k l}^{R}$ and $Z_{k l}^{I}$ for a fixed $k$ are $2 L$ i.i.d Gaussian random variables, each with zero mean and variance $E_{s} N_{0} / 2$. Therefore, the PDFs of $\Lambda_{1}$ and $\Lambda_{k}$ are given by, from [2] and [8] respectively

$$
\begin{aligned}
& p_{\Lambda_{1}}(\Lambda)=\sum_{l=1}^{L} \frac{B_{l}}{m_{l}} e^{-\frac{\Lambda}{m_{l}}}, \quad k=1 \\
& p_{\Lambda_{k}}(\Lambda)=\frac{\Lambda^{L-1}}{\left(E_{s} N_{0}\right)^{L}(L-1) !} e^{-\frac{\Lambda}{E_{s} N_{0}}}, k=2, \ldots, M,
\end{aligned}
$$

where,

$$
B_{l}=\prod_{i=1, i \neq l}^{L} \frac{1+\bar{\gamma}_{l}}{\bar{\gamma}_{l}-\bar{\gamma}_{i}}, \quad m_{l}=E_{s} N_{0}\left(\bar{\gamma}_{l}+1\right) .
$$

Applying the above PDFs to (9), the average SEP is obtained as

$$
\begin{aligned}
P_{s}(e) & =\sum_{m=1}^{M-1}\left(\begin{array}{c}
M-1 \\
m
\end{array}\right)(-1)^{m+1}\left[\frac{1}{(L-1) !}\right]^{m} \\
& \times \sum_{n_{1}, \ldots n_{L}} \frac{m !}{n_{1} ! \ldots n_{L} !} \prod_{i=1}^{L}\left[\frac{(L-1) !}{(L-i) !}\right]^{n_{i}}\left[\sum_{i=1}^{L} n_{i}(L-i)\right] ! \\
& \times \sum_{l=1}^{L} \frac{B_{l}}{1+m\left(1+\bar{\gamma}_{l}\right)}\left[\frac{1+\bar{\gamma}_{l}}{1+m\left(1+\bar{\gamma}_{l}\right)}\right]^{\sum_{i=1}^{L} n_{i}(L-i)}
\end{aligned}
$$

In the case where $L=2$, it can be shown that (16) agrees with the dual diversity case in [1, (20)]. Moreover, the result for binary FSK in $[2,(5)]$ becomes a special case of ours in (16) with $M=2$.

\section{SEP of Selection Combining SCHEMe}

Selection combining is another suboptimum diversity combining scheme. To date, there exist several selection criteria. The classic one is the the signal-to-noise ratio selection combining (SC-SNR), which selects the branch having the highest SNR [9]. Since the measurement of SNR may be difficult or expensive, maximum signal-plus-noise selection combining $(\mathrm{SC}-\mathrm{S}+\mathrm{N})$ is then often used [10]. The performance analysis of MFSK with SC-S+N over i.i.d Rayleigh fading channels can be found in [3]. Later, the analysis is extended by the authors in [4] to i.n.d channels. The SC-S+N scheme is, however, appropriate only if the average noise power is the same for all diversity branches [11]. Some researchers reexamined it and found that, for the practical implementation of $\mathrm{SC}-\mathrm{S}+\mathrm{N}$, the statistic behavior of output noise on the matched filter is different for different branches [10]. This observation leads to the scheme which selects the branch with the maximum output (SC-MO) at the demodulator. Authors in [3] analyzed the SC-MO performance for MFSK over i.i.d Rayleigh fading channel. Discussions over i.n.d Rayleigh fading channel is provided in [4]. Recently, SC-LLR was proposed in [5], [6] by selecting the branch providing the largest magnitude of loglikelihood ratio. The motivation of using LLR as the selection criterion is that it provides the information of the maximum a posteriori probability (MAP) based decision. Hence, this scheme is optimum among all selection combining schemes in the sense of minimizing the probability of symbol error. The SEP of MFSK with SC-LLR for i.i.d Rayleigh fading is obtained in [6] obtained. In this section, we extend the analysis to the general case where the $L$ diversity branches are i.n.d Rayleigh fading.

In SC-LLR, each diversity branch is equipped with a detector. Define

$$
\hat{m}_{l} \triangleq \arg \max _{m \in\{1, \ldots, M\}} P\left(\tilde{S}_{m}(t) \mid\left\{\left|y_{k l}\right|^{2}\right\}_{k=1}^{M}\right)
$$

as the MAP based estimate of the signal index on the $l$ th branch and $\left|y_{k l}\right|^{2}$ is the energy output of the lth branch after the $k$ th matched filter as shown in (2). Assuming that $\tilde{S}_{m}(t)$ was transmitted, we can define the LLR as

$$
\Lambda_{m l} \triangleq \ln \frac{P\left(\tilde{S}_{\hat{m}_{l}}(t) \mid\left\{\left|y_{k l}\right|^{2}\right\}_{k=1}^{M}\right)}{P\left(\tilde{S}_{m}(t) \mid\left\{\left|y_{k l}\right|^{2}\right\}_{k=1}^{M}\right)},
$$

for $m=1, \ldots, M$ and $l=1, \ldots, L$. Then $P\left(\tilde{S}_{\hat{m}_{l}}(t)\right.$ $\left.\left\{\left|y_{k l}\right|^{2}\right\}_{k=1}^{M}\right)$ can be rewritten as

$$
P\left(\tilde{S}_{\hat{m}_{l}}(t) \mid\left\{\left|y_{k l}\right|^{2}\right\}_{k=1}^{M}\right)=\frac{1}{\sum_{m=1}^{M} e^{-\Lambda_{m l}}} .
$$

Therefore, selecting the branch maximizing the posterior probability of $P\left(\tilde{S}_{\hat{m}_{l}}(t) \mid\left\{\left|y_{k l}\right|^{2}\right\}_{k=1}^{M}\right)$ is equal to choosing the branch minimizing $\sum_{m=1}^{M} e^{-\Lambda_{m l}}$. 
Since the signals are equally likely as assumed in Section II, the LLR $\Lambda_{m l}$ can also be expressed as

$$
\Lambda_{m l} \triangleq \ln \frac{P\left(\left\{\left|y_{k l}\right|^{2}\right\}_{k=1}^{M} \mid \tilde{S}_{\hat{m}_{l}}(t)\right)}{P\left(\left\{\left|y_{k l}\right|^{2}\right\}_{k=1}^{M} \mid \tilde{S}_{m}(t)\right)} .
$$

Similar to the discussion on (12) and (13), it can be shown that the variable $\left|y_{k l}\right|^{2}$ follows exponential distribution. The conditional PDF of $\left|y_{k l}\right|^{2}$ given the $m$ th signal $\tilde{S}_{m}(t)$ transmitted can thus be expressed as

$$
p_{\left|y_{k l}\right|^{2}}\left(\left|y_{k l}\right|^{2} \mid \tilde{S}_{m}(t)\right)=\left\{\begin{array}{cl}
\frac{1}{2 \sigma_{l}^{2}} e^{-\frac{\left|y_{k l}\right|^{2}}{2 \sigma_{l}^{2}}}, \quad k=m \\
\frac{1}{2 \sigma_{0}^{2}} e^{-\frac{\left|y_{k l}\right|^{2}}{2 \sigma_{0}^{2}}}, \quad k \neq m
\end{array}\right.
$$

where

$$
\sigma_{l}^{2}=E_{s} N_{0}\left(1+\bar{\gamma}_{l}\right) / 2, \quad \sigma_{0}^{2}=E_{s} N_{0} / 2 .
$$

Since $\left|y_{k l}\right|^{2}$ are independent for different $k$, the conditional joint PDF of $\left\{\left|y_{1 l}\right|^{2},\left|y_{2 l}\right|^{2}, \ldots,\left|y_{M l}\right|^{2}\right\}$ is obtained as

$$
\begin{aligned}
& P\left(\left\{\left|y_{k l}\right|^{2}\right\}_{k=1}^{M} \mid \tilde{S}_{m}(t)\right) \\
& =\left(\frac{1}{2 \sigma_{0}^{2}}\right)^{M-1}\left(\frac{1}{2 \sigma_{l}^{2}}\right) \exp \left(-\frac{\left|y_{m l}\right|^{2}}{2 \sigma_{l}^{2}}-\sum_{k=1, k \neq m}^{M} \frac{\left|y_{k l}\right|^{2}}{2 \sigma_{0}^{2}}\right)
\end{aligned}
$$

Similarly,

$$
\begin{aligned}
& P\left(\left\{\left|y_{k l}\right|^{2}\right\}_{k=1}^{M} \mid \tilde{S}_{\hat{m}_{l}}(t)\right) \\
& =\left(\frac{1}{2 \sigma_{0}^{2}}\right)^{M-1}\left(\frac{1}{2 \sigma_{l}^{2}}\right) \exp \left(-\frac{\left|y_{\hat{m}_{l} l}\right|^{2}}{2 \sigma_{l}^{2}}-\sum_{k=1, k \neq \hat{m}_{l}}^{M} \frac{\left|y_{k l}\right|^{2}}{2 \sigma_{0}^{2}}\right)
\end{aligned}
$$

By substituting (21) and (22) into (18), quantity $\Lambda_{m l}$ reduces to $[6,(32)]$

$$
\Lambda_{m l}=\left(\frac{1}{2 \sigma_{0}^{2}}-\frac{1}{2 \sigma_{l}^{2}}\right)\left(\left|y_{\hat{m}_{l} l}\right|^{2}-\left|y_{m l}\right|^{2}\right) .
$$

The estimate of the signal index on the lth branch $\hat{m}_{l}$ can be made by choosing the largest value of (21), since the MAP rule is equivalent to the maximum likelihood (ML) rule given equiprobable signals. By manipulating the exponential term of (21), it is seen that maximizing (21) is the same as maximizing term $\left(\frac{1}{2 \sigma_{0}^{2}}-\frac{1}{2 \sigma_{l}^{2}}\right)\left|y_{m l}\right|^{2}-\sum_{k=1}^{M} \frac{\left|y_{k l}\right|^{2}}{2 \sigma_{0}^{2}}$, and hence $\hat{m}_{l}=\arg \max _{m \in\{1, \ldots, M\}}\left|y_{m l}\right|^{2}$. Then, (17) can reduce to a simpler but suboptimal form

$$
P\left(\tilde{S}_{\hat{m}_{l}}(t) \mid\left\{\left|y_{k l}\right|^{2}\right\}_{k=1}^{M}\right) \leq \frac{1}{1+e^{-\left(\frac{1}{2 \sigma_{0}^{2}}-\frac{1}{2 \sigma_{l}^{2}}\right)\left(\left|y_{\hat{m}_{l} l}\right|^{2}-\left|y_{\hat{m}_{l} l}\right|^{2}\right)}}
$$

where, $\hat{\hat{m}}_{l}=\arg \max _{k \neq \hat{m}_{l}}\left|y_{k l}\right|^{2}$ is the signal index estimated on the $l$ th branch providing the second largest detector output. It is found from (24) that for i.n.d fading channels the branch with the maximum value of $\left(\frac{1}{\sigma_{0}^{2}}-\frac{1}{\sigma_{l}^{2}}\right)\left(\left|y_{\hat{m}_{l} l}\right|^{2}-\left|y_{\hat{\hat{m}}_{l} l}\right|^{2}\right)$ or equivalently $\frac{\bar{\gamma}_{l}}{1+\bar{\gamma}_{l}}\left(\left|y_{\hat{m}_{l} l}\right|^{2}-\left|y_{\hat{\hat{m}}_{l} l}\right|^{2}\right)$ for $l=1, \ldots L$ should be selected. So the decision metric in (23) is reduced to

$$
\Lambda_{m l}^{\prime}=\frac{\bar{\gamma}_{l}}{1+\bar{\gamma}_{l}}\left(\left|y_{\hat{m}_{l} l}\right|^{2}-\left|y_{\hat{\tilde{m}}_{l} l}\right|^{2}\right)
$$

We now readily derive the probability of symbol error of MFSK with the suboptimal SC-LLR scheme. Given that $\tilde{S}_{1}(t)$ was transmitted, the average SEP is given by

$$
P_{s}(e)=1-\sum_{j=1}^{L} P\left\{\text { branch } j \text { is selected and } \hat{m}_{j}=1\right\} \text {. }
$$

Here, we have

$$
\begin{aligned}
& P\left\{\text { branch } j \text { is selected and } \hat{m}_{j}=1\right\} \\
& =P\left\{\frac{\bar{\gamma}_{j}}{1+\bar{\gamma}_{j}}\left(\left|y_{1 j}\right|^{2}-\max _{k \neq 1}\left|y_{k j}\right|^{2}\right)>\right. \\
& \left.\quad \max _{l \neq j}\left\{\frac{\bar{\gamma}_{l}}{1+\bar{\gamma}_{l}}\left(\max _{k}\left|y_{k l}\right|^{2}-\max _{k \neq \hat{m}_{l}}\left|y_{k l}\right|^{2}\right)\right\}\right\} \\
& =\int_{0}^{\infty} P\left\{\frac{\bar{\gamma}_{j}}{1+\bar{\gamma}_{j}}\left(\left|y_{1 j}\right|^{2}-\max _{k \neq 1}\left|y_{k j}\right|^{2}\right)>r\right\} P_{\Gamma}(r) d r
\end{aligned}
$$

where $\Gamma \triangleq \max _{l \neq j}\left\{\frac{\bar{\gamma}_{l}}{1+\bar{\gamma}_{l}}\left(\max _{k}\left|y_{k l}\right|^{2}-\max _{k \neq \hat{m}_{l}}\left|y_{k l}\right|^{2}\right)\right\}$. The PDF of $\Gamma, P_{\Gamma}(r)$ is obtain as (28) at the bottom of the page. The proof is omitted for brevity. In (28), we have

$$
\begin{aligned}
& A_{l}=\sum_{k=0}^{M-1}\left(\begin{array}{c}
M-1 \\
k
\end{array}\right)(-1)^{k} \frac{1}{k\left(\bar{\gamma}_{l}+1\right)+1} \\
& B_{l}=(M-1) \sum_{k=0}^{M-2}\left(\begin{array}{c}
M-2 \\
k
\end{array}\right)(-1)^{k} \frac{1}{(k+1)\left[1+\left(1+\bar{\gamma}_{l}\right)(k+1)\right]} \\
& d_{i}=B_{i} e^{-\frac{\left(1+\bar{\gamma}_{i}\right) r}{2 \bar{\gamma}_{i}}}, \quad e_{i}=A_{i} e^{-\frac{r}{2 \bar{\gamma}_{i}}} .
\end{aligned}
$$

The closed-form expression for the SEP can thus be obtained as (29) shown at the top of the page by substituting (28) into (27) and (26), where $h_{n}$ for $n=0, \ldots i$ is given by (30). In (29), we have

$$
C_{l, j}=\frac{B_{l}}{1+\frac{\bar{\gamma}_{l}}{\bar{\gamma}_{j}\left(1+\bar{\gamma}_{l}\right)}}+\frac{A_{l}}{1+\frac{\bar{\gamma}_{l}}{\bar{\gamma}_{j}}} .
$$

In (30), we take $\prod_{k=1}^{n} A_{n_{p_{k}}}=1$ and $\sum_{i^{\prime}=n_{p_{1}}}^{n_{p_{n}}} \frac{1}{2 \bar{\gamma}_{i^{\prime}}}=0$ when $n<1$, and $\prod_{k=n+1}^{i} B_{n_{p_{k}}}=1$ and $\sum_{i^{\prime}=n_{p_{n+1}}}^{n_{p_{i}}} \frac{1+\bar{\gamma}_{i^{\prime}}}{2 \bar{\gamma}_{i^{\prime}}}=0$

$$
P_{\Gamma}(r)=\sum_{\substack{l=1 \\ l \neq j}}^{L}\left(\frac{\left(1+\bar{\gamma}_{l}\right) B_{l}}{2 \bar{\gamma}_{l}} e^{-\frac{\left(1+\bar{\gamma}_{l}\right) r}{2 \bar{\gamma}_{l}}}+\frac{A_{l}}{2 \bar{\gamma}_{l}} e^{-\frac{r}{2 \bar{\gamma}_{l}}}\right) \prod_{\substack{i=1 \\ i \neq l, j}}^{L}\left(1-d_{i}-e_{i}\right)
$$




$$
\begin{aligned}
& P_{s}(e)=1-\sum_{j=1}^{L} A_{j} \sum_{\substack{l=1 \\
l \neq j}}^{L} C_{l, j}+\sum_{i=1}^{L-2} \sum_{\substack{n_{1}, n_{2}, \ldots, n_{i}=1 \\
n_{1}, n_{2}, \ldots, n_{i} \neq l, j \\
n_{1}<n_{2}<\ldots<n_{i}}}^{L}\left(\sum_{\substack{p_{1}, p_{2}, \ldots, p_{i}=1 \\
p_{1}<p_{2}<\ldots<p_{i}}}^{i} h_{0}+\sum_{\substack{p_{1}, p_{2}, \ldots, p_{i}=1 \\
p_{2}, p_{3}, \ldots, p_{i} \neq p_{1} \\
p_{2}<p_{3}<\ldots<p_{i}}}^{i} h_{1}+\ldots+\sum_{\substack{p_{1}, p_{2}, \ldots, p_{i}=1 \\
p_{1}<p_{2}<\ldots<p_{i}}}^{i} h_{i}\right) \\
& h_{n}=\frac{B_{l} \prod_{k=1}^{n} A_{n_{p_{k}}} \prod_{k=n+1}^{i} B_{n_{p_{k}}}^{i}}{\frac{2 \bar{\gamma}_{l}}{1+\bar{\gamma}_{l}}\left(\frac{\bar{\gamma}_{l}+\bar{\gamma}_{j}\left(1+\bar{\gamma}_{l}\right)}{2 \bar{\gamma}_{j} \bar{\gamma}_{l}}+\sum_{i^{\prime}=n_{p_{1}}}^{n_{p_{n}}} \frac{1}{2 \bar{\gamma}_{i^{\prime}}}+\sum_{i^{\prime}=n_{p_{n+1}}}^{n_{p_{i}}} \frac{1+\bar{\gamma}_{i^{\prime}}}{2 \bar{\gamma}_{i^{\prime}}}\right)}+\frac{A_{l} \prod_{k=1}^{n} A_{n_{p_{k}}} \prod_{k=n+1}^{i} B_{n_{p_{k}}}}{2 \bar{\gamma}_{l}\left(\frac{\bar{\gamma}_{l}+\bar{\gamma}_{j}}{2 \bar{\gamma}_{j} \bar{\gamma}_{l}}+\sum_{i^{\prime}=n_{p_{1}}}^{n_{p_{n}}} \frac{1}{2 \bar{\gamma}_{i^{\prime}}}+\sum_{i^{\prime}=n_{p_{n+1}}}^{n_{p_{p_{i}}}} \frac{1+\bar{\gamma}_{i^{\prime}}}{2 \bar{\gamma}_{i^{\prime}}}\right)} .
\end{aligned}
$$

when $i<n+1$ respectively. Even though our result in (29) is for the more general i.n.d channels, the SEP for i.i.d channels can be obtained directly by letting $\bar{\gamma}_{l}=\bar{\gamma}, \forall l$, which agrees with $[6,(45)]$.

\section{NUMERICAL RESUlTS AND DISCUSSION}

In this section, we present numerical examples to compare the performance of OC, EGC, and different selection combining schemes. An exponentially decaying power profile characterized by $\bar{\gamma}_{l}=\bar{\gamma}_{1} e^{-\delta(l-1)}$, for $1 \leq l \leq L$, is used, in which $\delta \geq 0$ is the average power decay factor. A larger value of $\delta$ indicates higher degree of unbalance of the non-identical fading channels.

Fig. 1 shows the average SEP of BFSK versus the average SNR of the first branch $\bar{\gamma}_{1}$, for $\delta=1$ with diversity order $L=2$ and 4 . Note that the results of SC-SNR and SC-MO are from [4]. It is seen that the performance of EGC is almost identical to that of the OC scheme. One can also see that SCLLR performs the best out of all the three selection combining schemes and that its performance loss compared with OC is negligible when $L=2$.

Fig. 2 compares the SEP performance among the five combining schemes with $\delta=1,3$ and 5 for modulation level $M=4$ and diversity order $L=4$. It is again seen that SC-LLR is always superior to SC-MO and SC-SNR. Yet, EGC is not always the one that performs the most closely to OC. In particular, for slightly unbalanced channels $(\delta=1)$, EGC has almost the same performance as OC, but for heavily unbalanced channels $(\delta=5)$, its performance degrades. On the contrary, SC-LLR for heavily unbalanced channels becomes the best out of all suboptimal combining schemes and the performance gap to the optimal combining is only marginal. These observations can be explained as follows. For slightly unbalanced channels, the difference between the optimal weights applied in OC is insignificant and thus simply applying equal weights on each branch (i.e. EGC) would result in similar performance. Unlike EGC, the selection combining, which makes use of one branch only, is much more likely to make an erroneous detection when the diversity branches experience deep fading simultaneously, and hence performs worse than EGC does. For highly unbalanced channels, since the optimal weight applied to each branch in OC differs significantly from each other, it is not appropriate to approximate $\mathrm{OC}$ by applying equal weights. Furthermore, as the branches are highly unbalanced,

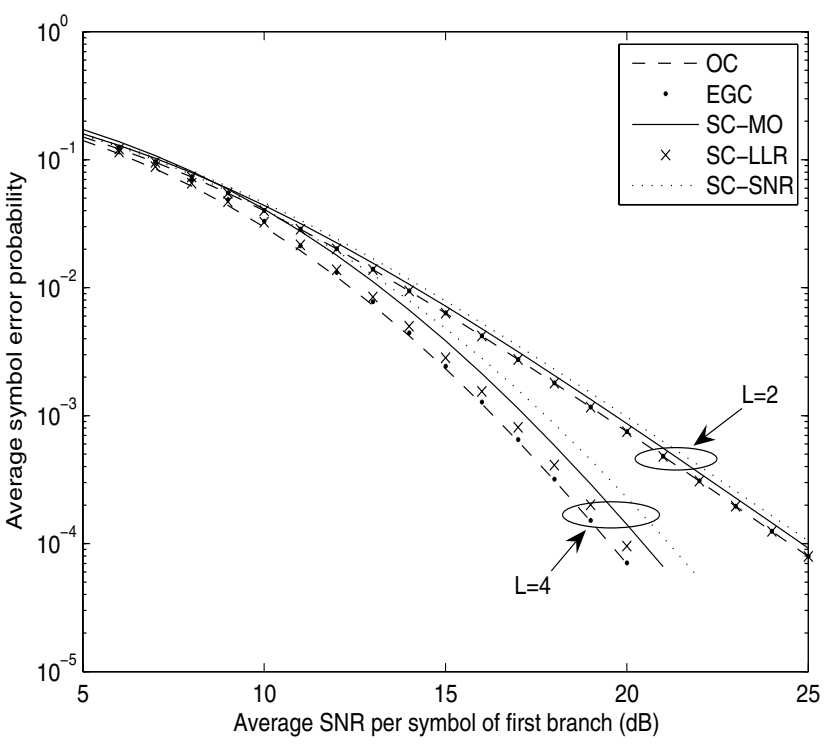

Fig. 1. Average SEP performance of BFSK over $L$-branch diversity with power decay factor $\delta=1$

the probability of having all branches in deep fade becomes much smaller and consequently, SC-LLR can always find the best branch and outperforms EGC.

In addition to error performance, the implementation complexity is also of great concern. Here, we take the outputs of the square-law envelop detectors $\left|y_{k l}\right|^{2}$, and compute the number of numerical operations per symbol detection. Through evaluation of (3), (11) and (25), we form Table I which shows the number of operations needed with OC, EGC and SC-LLR. By considering the implementation complexity of $\mathrm{OC}$ as the benchmark, it is shown from Table I that EGC can save $M L$ multiplications per symbol detection. On the other hand, SCLLR can save $(M-1) L$ multiplications and $M(L-1)-L$ additions but at the cost of $M(L-1)$ more comparisons per symbol detection.

\section{CONCLUSIONS}

We presented the closed-form expressions for symbol error probability of noncoherent orthogonal MFSK with OC, EGC, and SC-LLR diversity receptions over independent and nonidentically distributed Rayleigh fading channels. Our results 


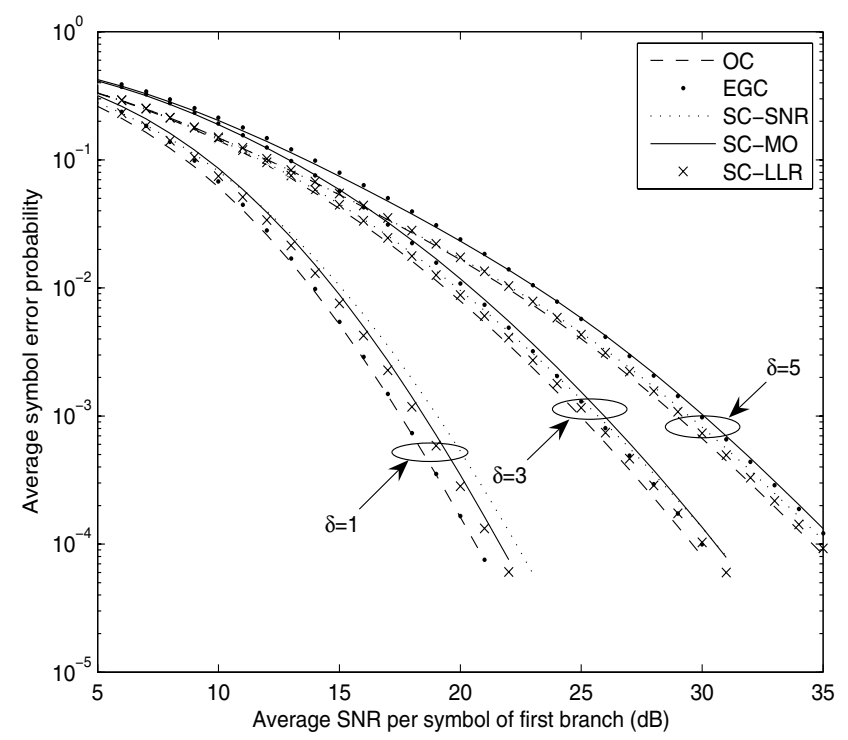

Fig. 2. Average SEP performance of 4FSK over 4-branch diversity with power decay factor $\delta=1,3$ and 5

TABLE I

COMPARISON ON COMPUTATIONAL COMPLEXITY PER SYMBOL DETECTION.

\begin{tabular}{|c|c|c|c|}
\hline Operation & Addition & Comparison & Multiplication \\
\hline EGC & $M(L-1)$ & $M-1$ & 0 \\
\hline OC & $M(L-1)$ & $M-1$ & $M L$ \\
\hline SC-LLR & $L$ & $L M-1$ & $L$ \\
\hline
\end{tabular}

are by far the most complete ones in the sense that they are for arbitrary level of modulations and arbitrary number of diversity branches. Analytical performance comparison among the different diversity combining schemes was made under various channel and modulation parameters. In general we observed that among all the suboptimal combining schemes, EGC has the most close performance to OC when the diversity branches are slightly unbalanced. Yet, for heavily unbalanced channels, the SC-LLR scheme becomes more superior and it has only marginal performance loss compared with OC. We also found that, compared with OC, SC-LLR can save significant implementation complexity in the number of multiplications and additions per symbol detection, even though it needs more number of comparisons.

\section{ACKNOWLEDGMENT}

The authors would like to thank Dr. H. Fu for fruitful discussions.

\section{REFERENCES}

[1] M. K. Simon and M. S. Alouini, "Average bit-error probability performance for optimum diversity combining of noncoherent FSK over Rayleigh fading channels," IEEE Trans. Commun., vol. 51, No. 4, Apr. 2003.

[2] D. Torrieri, "Simple formular for error probability of Rayleigh fading," IEEE Trans. Commun., Vol. 50, pp. 1734-1735, Nov. 2002.
[3] G. Chyi, J. G. Proakis and C. M. Keller, "On the symbol error probability of maximum-selection diversity reception schemes over Rayleigh fading channel," IEEE Trans. Commun., vol. 37, pp. 79-83, Jan. 1989.

[4] S. Haghani, Norman C. Beaulieu, "Symnol Error Rate Performance of M-ary NCFSK with S+N Selection Combining in Rician Fading," Proc. IEEE ICC, vol. 4, pp. 2500-2505, May, 2005.

[5] Y. G. Kim and S. W. Kim, "Optimum selection diversity for BPSK signals in Rayleigh fading channels," IEEE Trans. Commun., vol. 49, pp. 1715-1718, Oct. 2001.

[6] Y. G. Kim and S. W. Kim, "Optimum selection combining for Mary signals in frequency-nonselective fading channels," IEEE Trans. Commun., vol. 53, Jan. 2005.

[7] M. K. Simon and M. S. Alouini, Digital Communications Over Fading Channels. New York: Wiley, 2000.

[8] J. G. Proakis, Digital Communications. 4th ed. New York: McGrawHill, 2001.

[9] G. L. Stuber, Principle of Mobile Communication. Norwell: MA Kluwer, 1996

[10] E. A. Neasmith and N. C. Beaulieu, "New Results on Selection Diversit," IEEE Trans. Commun., vol. 46, pp. 695-704, May, 1998.

[11] F. E. Bond and H. F. Meyer, "The effect of fading on communication circuits subject to interference," Proc. IRE, vol. 45, pp. 636-642, May, 1957. 